[RAdiocarbon, Vol. 14, No. 2, 1972, P. 486-497]

\title{
UNIVERSITY OF GEORGIA RADIOCARBON DATES II
}

\author{
BETTY LEE BRANDAU and JOHN E. NOAKES \\ Geochronology Laboratory \\ University of Georgia, Athens, Georgia 30601
}

The following list of dates is compiled from samples prepared since publication of our last date list (R., 1971, v. 13, p. 468-474). The counting equipment and operating procedures are the same. Ages are quoted with a $1 \sigma$ counting error which includes statistical variation of the sample count as well as for the background and standard, using A.D. 1950 as the reference year and $0.95 \%$ NBS oxalic acid for $\mathrm{C}^{14}$ dating as the standard. The half-life value used is 5570 years.

Sample descriptions were prepared in collaboration with collectors and submitters.

\section{ACKNOWLEDGMENTS}

The support of the General Research Department of the University of Georgia is gratefully acknowledged. Donald F. Smith has prepared many of the samples described in this list.

\section{SAMPLE DESCRIPTIONS}

I. GEOLOGIC SAMPLES

\section{A. Georgia}

The following samples are from cores in the low-lying marshlands around Sapelo I., Georgia. They are part of a study, including pollen analysis, being made by Joyce Swanberg. After removal from cores, sediments were wrapped in polyethylene and brought to the lab where the outer surfaces were cut away. Samples were then dried and burned, so the date is a composite of total combustible carbon.

Core 5ABBS from salt marsh between Blackbeard and Sapelo Is. on $\mathrm{W}$ side of Blackbeard Creek (31 $31^{\prime} 5^{\prime \prime} \mathrm{N}$ Lat, $81^{\circ} 13^{\prime} 10^{\prime \prime} \mathrm{W}$ Long).

UGa-195. 81 to $89 \mathrm{~cm}$

UGa-91. 152 to $164 \mathrm{~cm}$

UGa-133. 479 to $493 \mathrm{~cm}$

Core $5 \mathrm{BBS}, 100 \mathrm{~m} \mathrm{~S}$ of $5 \mathrm{ABBS}$.

UGa-188. 151 to $161 \mathrm{~cm}$

UGa-187. 242 to $253 \mathrm{~cm}$

UGa-207. 336 to $352 \mathrm{~cm}$
$1550 \pm 80$

A.D. 400

$2090 \pm 500$

140 B.C.

$3445 \pm 140$

1495 в.C.

$$
1550 \pm 80
$$

A.D. 400

$$
1110 \pm 70
$$

A.D. 840

$1140 \pm 100$ A.D. 810 
UGa-127. 353 to $362 \mathrm{~cm}$

$$
1130 \pm 105
$$

Core 4BBS from salt marsh between Blackbeard and Sapelo Is. on W side of Blackbeard Creek ( $31^{\circ} 30^{\prime} 45^{\prime \prime}$ N Lat, $8^{\circ} 13^{\prime} 0^{\prime \prime}$ W Long).

UGa-216. 173 to $180 \mathrm{~cm}$

UGa-138. 226 to $270 \mathrm{~cm}$

UGa-171. 315 to $320 \mathrm{~cm}$

UGa-209. 384 to $391 \mathrm{~cm}$

UGa-169. 425 to $432 \mathrm{~cm}$

UGa-137. 445 to $453 \mathrm{~cm}$

UGa-106. 514 to $521 \mathrm{~cm}$
$1380 \pm 95$ A.D. 570

A.D. 1490

$$
460 \pm 130
$$

$1040 \pm 100$ A.D. 910

A.D. 910

$$
1040 \pm 90
$$

$1040 \pm 100$ A.D. 910

$$
1215 \pm 100
$$

A.D. 735

$$
730 \pm 100
$$

A.D. 1220

Core 9BBS, SW of Lookout Tower of Blackbeard Island Refuge (31 $29^{\prime} 0^{\prime \prime} \mathrm{N} \mathrm{Lat,} 81^{\circ} 13^{\prime \prime} 0^{\prime \prime} \mathrm{W}$ Long).

UGa-184. 155 to $164 \mathrm{~cm}$

$2220 \pm 100$ 270 B.c.

UGa-177. 291 to $302 \mathrm{~cm}$ $2180 \pm 110$ 230 B.C.

UGa-126. 454 to $468 \mathrm{~cm}$ $2440 \pm 330$ 490 в.c.

Core 2ATCS salt marsh between Old Teakettle Creek and New Teakettle Creek, Sapelo I. (31 $28^{\prime} 0^{\prime \prime} \mathrm{N}$ Lat, $81^{\circ} 18^{\prime} 20^{\prime \prime} \mathrm{W}$ Long).

UGa-155. 80 to $90 \mathrm{~cm}$ A.D. 1130

$820 \pm 95$

UGa-154. 138 to $147 \mathrm{~cm}$ $3900 \pm 125$ 1350 B.C.

UGa-160. 349 to $356 \mathrm{~cm}$ $4450 \pm 190$ 2500 в.C.

UGa-202. 400 to $414 \mathrm{~cm}$ $4350 \pm 135$ 2400 B.C.

UGa-121. 421 to $430 \mathrm{~cm}$ $3900 \pm 170$ 1950 B.c.

Core 1ATCS, $270 \mathrm{~m}$ NW of Jack Hammock between New Teakettle Creek and Duplin R. (31 $27^{\prime} 50^{\prime \prime} \mathrm{N}$ Lat, $81^{\circ} 17^{\prime} 35^{\prime \prime} \mathrm{W}$ Long). 
UGa-189. 116 to $123 \mathrm{~cm}$

UGa-175. 222 to $230 \mathrm{~cm}$

UGa-165. 334 to $342 \mathrm{~cm}$

UGa-123. 410 to $419 \mathrm{~cm}$

Core 8 POC, from salt marsh between Post Office Creek and Little Sapelo I. (31 $26^{\prime} 2^{\prime \prime} \mathrm{N} \mathrm{Lat,} 81^{\circ} 17^{\prime} 30^{\prime \prime} \mathrm{W}$ Long).

UGa-211. 427 to $435 \mathrm{~cm}$

UGa-194. 567 to $576 \mathrm{~cm}$

UGa-132. 708 to $718 \mathrm{~cm}$

UGa-128. 784 to $798 \mathrm{~cm}$

UGa-117. Shell $436 \mathrm{~cm}$

Core 6DCS, from salt marsh near Dark Creek $\left(31^{\circ} 27^{\prime} 6^{\prime \prime}\right.$ N $81^{\circ} 19^{\prime} 22^{\prime \prime}$ W Long).

UGa-153. 62 to $70 \mathrm{~cm}$

UGa-162. 142 to $150 \mathrm{~cm}$

UGa-156. 178 to $190 \mathrm{~cm}$

Core 3ATCS, from salt marsh between Atwood Creek and Old Teakettle Creek ( $31^{\circ} 28^{\prime} 0^{\prime \prime} \mathrm{N}$ Lat, $81^{\circ} 20^{\prime} 0^{\prime \prime} \mathrm{W}$ Long).

UGa-180. 134 to $143 \mathrm{~cm}$

UGa-178. 252 to $260 \mathrm{~cm}$

UGa-136. 359 to $368 \mathrm{~cm}$

Comment: depth-age relationship of some cores leaves much to be desired; perhaps marsh sediments were reworked more than expected.

The following shell and wood samples from vicinity of Marine Inst., Sapelo I., Georgia, as part of a continuing investigation of the region. Samples coll. and subm. by H. U. Wiedemann, Dept. Geol., Univ. Georgia.

A.D. 1590

$$
3440 \pm 125
$$$$
1490 \text { B.c. }
$$$$
4470 \pm 195
$$$$
2520 \text { B.C. }
$$

$$
\begin{gathered}
1270 \pm 85 \\
\text { A.D. } 680 \\
2430 \pm 100 \\
480 \text { B.c. } \\
2425 \pm 170 \\
475 \text { B.c. }
\end{gathered}
$$

$3150 \pm 115$

$3190 \pm 90$

$3115 \pm 115$

$3250 \pm 125$

1300 в.C. (a) 
UGa-87. Dark Creek oyster

$1190 \pm 65$

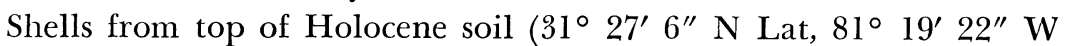
Long), from $30 \mathrm{~cm}$ deep oyster bed. Valves articulated; hence not reworked.

UGa-88. Atwood Creek wood

$2400 \pm 75$

450 B.C.

Wood from tree trunks in submerged early Holocene soil, $\left(31^{\circ}\right.$ $28^{\prime} 6^{\prime \prime} \mathrm{N}$ Lat, $81^{\circ} 20^{\prime} 44^{\prime \prime} \mathrm{W}$ Long), depth ca. $30 \mathrm{~cm}$ ( $2 \mathrm{~m}$ below surface of present marsh).

UGa-90. Teakettle Creek wood

$2450 \pm 75$

500 B.C.

Wood from tree trunks in submerged early Holocene soil, depth $50 \mathrm{~cm},\left(31^{\circ} 27^{\prime} 6^{\prime \prime} \mathrm{N}\right.$ Lat, $81^{\circ} 19^{\prime} 22^{\prime \prime} \mathrm{W}$ Long).

\section{UGa-93. Blackbeard Creek Marsh oyster}

$1640 \pm 150$

Shell from basal $15 \mathrm{~cm}$ of bed $5.5 \mathrm{~m}$ below marsh surface $\left(31^{\circ} 31^{\prime} 5^{\prime \prime}\right.$ $\mathrm{N}$ Lat, $81^{\circ} 13^{\prime} 10^{\prime \prime} \mathrm{W}$ Long). Shells blackened by $\mathrm{FeS}_{2}$ and bored by Clinona.

\section{UGa-95. Blackbeard Creek Marsh oyster}

$$
1720 \pm 90
$$

Articulated shell from $2.8 \mathrm{~m}$ below marsh surface. Core near UGa93 but shells not blackened or bored.

\section{UGa-97. Todd Creek Marsh oyster}

$1260 \pm 75$

Shell from tidal chenier on marsh surface, $\left(31^{\circ} 32^{\prime} 50^{\prime \prime} \mathrm{N}\right.$ Lat, $81^{\circ}$ $13^{\prime} 16^{\prime \prime}$ W Long).

\section{UGa-99. Todd Creek Marsh oyster}

$580 \pm 120$

Location slightly SW of UGa-97 $\left(31^{\circ} 32^{\prime} 45^{\prime \prime} \mathrm{N}\right.$ Lat, $81^{\circ} 13^{\prime} 25^{\prime \prime}$ W Long) similar occurrence.

\section{UGa-98. Todd Creek Hammock oyster}

$390 \pm 120$

Shell from low hammock, perhaps a former beach ridge. Presently covered by modern tidal-marsh soil $\left(31^{\circ} 33^{\prime} 4^{\prime \prime} \mathrm{N} \mathrm{Lat,} 81^{\circ} 13^{\prime} 30^{\prime \prime} \mathrm{W}\right.$ Long).

UGa-107. Teakettle Creek Marsh oyster

$730 \pm 120$

Shell from another chenier $60 \mathrm{~m} \mathrm{E}$ of UGa-110 and $115 \mathrm{~m} \mathrm{~N}$ of present margin of sound ( $31^{\circ} 25^{\prime} 55^{\prime \prime} \mathrm{N}$ Lat, $81^{\circ} 19^{\prime} 10^{\prime \prime} \mathrm{W}$ Long).

UGa-109. Doboy Sound shell

$135 \pm 185$

Oyster and shell hash from $\mathrm{E}$ bank of Doboy Sound at mouth of Duplin R. (31 $24^{\prime} 43^{\prime \prime} \mathrm{N}$ Lat, $81^{\circ} 17^{\prime} 58^{\prime \prime} \mathrm{W}$ Long), $80 \mathrm{~cm}$ depth at 
base of oyster concentrate overlyinig mud. Bank presently undergoing erosion.

\section{UGa-111. Shell Hammock Marsh shell}

$$
1380 \pm 195
$$

Oyster hash from small shell chenier in marsh off Shell Hammock, Sapelo I. ca. $80 \mathrm{~m}$. from island next to dike around Reynolds Marsh (31 $21^{\prime} 57^{\prime \prime} \mathrm{N}$ Lat, $81^{\circ} 17^{\prime} 11^{\prime \prime} \mathrm{W}$ Long). Shells from $1 \mathrm{~m}$ depth in gray marsh mud next to chenier on its landward side, in top $1.5 \mathrm{~m}$ of sediment.

\section{UGa-100. Shell Hammock oyster-NW}

$$
1590 \pm 420
$$

\section{A.D. 360}

Shell from depth $75 \mathrm{~cm}$ at contact of shell layer with underlying marsh peat ( $31^{\circ} 24^{\prime} 19^{\prime \prime} \mathrm{N}$ Lat, $81^{\circ} 17^{\prime} 33^{\prime \prime} \mathrm{W}$ Long). Formation indicates former sound margin.

\section{UGa-102. Shell Hammock oyster-SE}

$$
70 \pm 125
$$

SE of UGa-100 with similar surroundings ( $31^{\circ} 24^{\prime} 15^{\prime \prime} \mathrm{N} \mathrm{Lat,} 81^{\circ} 17^{\prime}$ 32" W Long). Comment (H.U.W.): such a recent date seems unlikely.

\section{UGa-103. Black River Marsh oyster}

Shell from low chenier in marsh $\mathrm{S}$ of Back R., where it merges

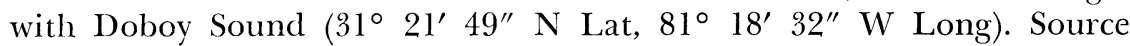
$180 \mathrm{~m}$ from sound in marsh interior from depth $75 \mathrm{~cm}$ at contact of shell deposit and underlying marsh.

\section{UGa-110. Teakettle Creek Marsh oyster}

Shell from low chenier in marsh $\mathrm{N}$ of point where creek merges with Doboy Sound, $60 \mathrm{~m} \mathrm{~N}$ of present sound margin.

\section{Georgia Continental Shelf series}

Shells from box cores on continental shelf off Georgia were dated to study movement and deposition of coastal sediment.

\section{UGa-232. S-72}

$$
1750 \pm 115
$$

Argopectin gibbus, Pecten raveneli, Laevicardium pictum from upper 0.3 to $0.6 \mathrm{~m}$ sediment under $26 \mathrm{~m}$ water $\left(31^{\circ} 4^{\prime} 30^{\prime \prime} \mathrm{N}\right.$ Lat, $80^{\circ}$ 32' 12" W Long).

UGa-230. S-60

Modern

Argopectin gibbus from upper 0.3 to $0.6 \mathrm{~m}$ sediment under $49 \mathrm{~m}$ water ( $31^{\circ} 14^{\prime} 36^{\prime \prime} \mathrm{N}$ Lat, $79^{\circ} 57^{\prime} 0^{\prime \prime} \mathrm{W}$ Long).

\section{UGa-231. S-45}

Crassostrea virginica from upper 0.3 to $0.6 \mathrm{~m}$ sediment beneath $18 \mathrm{~m}$ water $\left(31^{\circ} 23^{\prime} 0^{\prime \prime} \mathrm{N}\right.$ Lat, $80^{\circ} 48^{\prime} 0^{\prime \prime} \mathrm{W}$ Long). 
Dosinia elegans from upper 0.3 to $0.6 \mathrm{~m}$ sediment under $47.5 \mathrm{~m}$ water ( $31^{\circ} 31^{\prime} 42^{\prime \prime} \mathrm{N}$ Lat, $79^{\circ} 53^{\prime} 12^{\prime \prime} \mathrm{W}$ Long).

Shells coll. by J. D. Howard and R. W. Frey; subm. by B. K. Sen Gupta, Geol. Dept., Univ. Georgia. Comment (B.S.G.): diversity of ages suggests greater transport of heavy shell material than previously thought.

\section{Alluvial Wood series}

Samples of wood recovered from alluvial terrace of Savannah R. at Merry Bros. Brick \& Tile Co., Plant \#1, Augusta, Georgia (33 26' $\mathrm{N}$ Lat, $81^{\circ} 56^{\prime} \mathrm{W}$ Long).

UGa-173. $6 \mathrm{~m}$ below surface $>40,000$

UGa-174. ) $>40,000$

UGa-172. $\} 10$ to $11 \mathrm{~m}$ below surface $>40,000$

$\begin{array}{rr}\text { UGa-161. } & >40,000 \\ \text { UGa-170. } & 35,000 \pm 2300\end{array}$ 33,050 в.C.

Coll. by R. E. Carver, Dept. Geol., Univ. Georgia. Comment (B.L.B.): ages, unfortunately, near or beyond range of $\mathrm{C}^{14}$ dating.

\section{Giant Sloth Bone series}

From $11.2 \mathrm{~km} \mathrm{NW}$ of Brunswick, Glynn Co., Georgia $\left(31^{\circ} 14^{\prime} \mathrm{N}\right.$ Lat, $81^{\circ} 29^{\prime} \mathrm{W}$ Long). Coll. by Michael Voorhees and Albert Brantley, Dept. Geol., Univ. Georgia.

\section{UGa-79. Bone}

$$
\mathbf{9 3 8 0} \pm 85
$$

\section{UGa-80. Bone}

$11,310 \pm 90$

UGa-79 is inner porous bone material and UGa-80 is the date of the outer dense bone material. Samples were rinsed in distilled water, crushed, soaked in $1 \mathrm{~N} \mathrm{HCl}$ and rinsed. The carbon recovered from the dry bone in burning was ca. $0.5 \%$ total sample weight. From area of many bones and whole skeletons from several extinct species.

\section{Amelia Island shell series}

\section{B. Florida}

Shells from Amelia I., Florida, from cores at various locations to help determine chronology of formation of island.

\section{UGa-218.}

Shell fragments in fine gray sand, ca. $12 \mathrm{~m}$ from surface $\left(30^{\circ} 35^{\prime}\right.$ 25" N Lat, 81 26' 55" W Long). Small sample.

UGa-219.

Shell fragments in mud ca. $15 \mathrm{~m}$ from surface $\left(30^{\circ} 34^{\prime} 10^{\prime \prime} \mathrm{N}\right.$ Lat, $81^{\circ} 27^{\prime} 6^{\prime \prime}$ W Long). 
UGa-217.

Shell fragments in fine sand and silt ca. $14 \mathrm{~m}$ from surface $\left(30^{\circ}\right.$ $33^{\prime} 29^{\prime \prime} \mathrm{N}$ Lat, $81^{\circ} 27^{\prime} 0^{\prime \prime} \mathrm{W}$ Long).

\section{UGa-214.}

$$
4950 \pm 70
$$

Fine shell hash in silty fine sand ca: $13 \mathrm{~m}$ below $\operatorname{surface}\left(30^{\circ} 33^{\prime}\right.$ $40^{\prime \prime} \mathrm{N}$ Lat, $81^{\circ} 26^{\prime} 36^{\prime \prime}$ W Long).

\section{UGa-221.}

Shell fragments, mostly oyster, from $5 \mathrm{~m}$ below surface $\left(30^{\circ} 31^{\prime}\right.$ $51^{\prime \prime} \mathrm{N}$ Lat, $81^{\circ} 26^{\prime} 52^{\prime \prime} \mathrm{W}$ Long).

\section{UGa-222.}

Shell fragments in mud 2 to $3 \mathrm{~m}$ below surface $\left(30^{\circ} 31^{\prime} 33^{\prime \prime} \mathrm{N}\right.$ Lat, $81^{\circ} 26^{\prime} 33^{\prime \prime} \mathrm{W}$ Long). Samples subm. by V. J. Henry, Marine Inst., Sapelo I., Georgia.

$$
\text { C. Colombia, South America }
$$

\section{Ciénaga Grande series}

Samples coll. as part of investigation of lagoon formation.

UGa-152. Ciénaga Grande 16 peat (10 $57^{\prime} 48^{\prime \prime} \mathrm{N}$ Lat, $74^{\circ} 19^{\prime} 42^{\prime \prime}$ W Long)

UGa-149. Ciénaga Grande 127 peat (10 $54^{\prime} 48^{\prime \prime} \mathrm{N}$ Lat, $74^{\circ} 24^{\prime} 6^{\prime \prime} \mathrm{W}$ Long)

UGa-151. Ciénaga Grande 138 peat ( $10^{\circ} 48^{\prime} 36^{\prime \prime} \mathrm{N}$ Lat, $74^{\circ} 26^{\prime} 23^{\prime \prime} \mathrm{W}$ Long)

UGa-150. Ciénaga Grande 119 peat (10 $46^{\prime} 6^{\prime \prime} \mathrm{N}$ Lat, $74^{\circ} 24^{\prime} 6^{\prime \prime} \mathrm{W}$ Long)

\section{UGa-146. Ciénaga Grande 118 oyster} (10 $45^{\prime} 0^{\prime \prime} \mathrm{N}$ Lat, $74^{\circ} 24^{\prime} 6^{\prime \prime}$ W Long)

Samples from lagoon separated from Caribbean Sea by narrow sand barrier. Lagoon uniformly $2 \mathrm{~m}$ deep. Peat indicates a rising sea level beginning ca. $2400 \mathrm{yr}$ ago and encroaching from N. Shell is from more recent estuarine sediments overlying peat. Samples coll., subm. and commented on by H. U. Wiedemann.
$2430 \pm 85$ 480 в.c.

$2300 \pm 65$ 350 B.C.

$$
1920 \pm 65
$$

A.D. 30

$$
1920 \pm 65
$$

A.D. 30

$$
\text { A.D. } 1670
$$

$$
280 \pm 80
$$


II. ARGHAEOLOGIC SAMPLES

A. South

1. Mississippi

Boyd site series

Boyd site 22-Tu-531, Tunica Co., Mississippi (34 $36^{\prime} \mathrm{N}$ Lat, $90^{\circ}$ $25^{\prime} 10^{\prime \prime}$ W Long).

UGa-159. Charcoal

Level 0.6 to $0.8 \mathrm{~m}$. Feature \#28, refuse pit.

\section{UGa-163. Charcoal}

Level 0.4 to $1.4 \mathrm{~m}$. Feature \#22, refuse pit.

\section{UGa-158. Charcoal}

Level 0.6 to $0.8 \mathrm{~m}$. Feature \#10, refuse pit.

\section{UGa-164. Charcoal}

Lower Midden, below UGa-158, Feature \#37, refuse pit.

\section{UGa-166. Charcoal}

Lower midden, below U( Ga-158, Feature \#37, refuse pit.
$2170 \pm 90$

$1410 \pm 70$

A.D. 540

$1500 \pm 75$

A.D. 450

$1700 \pm 80$

A.D. 250

$1865 \pm 100$

A.D. 85

220 B.c.

firment: dates confirm range anticipated from ceramic analysis. The sequence in which they occur also appears good. Dates represent 2 components separated by a layer of sterile sand. The lower component is of the Tchula period, the upper is late Markville and Baytown. The earlier dates, 220 B.c. and A.D. 85 are from the lower stratum. The 3 younger dates are from the upper stratum and fall into correct order of age based on ceramic seriation.

Clear Creek site 22-La-542, Lafayette Co., Mississippi $\left(34^{\circ} 25^{\prime} 5^{\prime \prime}\right.$ N Lat, $89^{\circ} 42^{\prime} 48^{\prime \prime}$ W Long).

\section{UGa-167. Charcoal}

$$
1620 \pm 90
$$

Cultural assoc. is Tchula and Early Baytown; date seems inconsistent with ceramic assoc. which would tend to place it in interval between the 2 occupation zones at Boyd site, A.D. 85 to A.D. 250.

Denton site 22-Qu-522, Quitman Co., Mississippi (34 $9^{\prime} 4^{\prime \prime} \mathrm{N}$ Lat, $90^{\circ} 19^{\prime} 26^{\prime \prime}$ W Long).

\section{UGa-212. Charcoal}

$5230 \pm 125$ 3280 B.c.

0.3 to $0.5 \mathrm{~m}$ below surface. Site was thought to be pre-Poverty point because of surface collections. Date confirms belief but is somewhat earlier than expected. The Denton excavation was the 1st of an Archaic, pre-Poverty Point site in the Yazoo Basin to yield a date. Similar sites 
are now being considered for testing and should elucidate significance of date.

Mississippi samples coll. and commented on by John Connaway and Sam McGahey, State Survey Archaeologists.

\section{Georgia}

\section{Table Point site series}

Table Point site, Cumberland I., Georgia (30 52' 30" N Lat, $81^{\circ}$ $28^{\prime} 0^{\prime \prime}$ W Long).

\section{UGa-129. Busycon perversum (linné)}

$$
1895 \pm 95
$$

Shell from a house pattern of Deptford period, shows use-battering on tip. $80 \%$ of assoc. pottery is Deptford with some fiber-tempered and semi-fiber tempered pottery, placing date early in Deptford period. Date pinpoints time of emergence from (or intrusion into) Transitional period by the Deptford peoples on the SE Georgia coast.

UGa dates are 1st for Deptford on Georgia Coast, previous dates were intuitive. It now seems that populations were smaller, diffusion slower, and cultural changes less rapid on Georgia coastal plain than supposed. Sample coll. and comment by Jerald Melanich, Dept. Archaeol., Univ. Florida.

UGa-140. 9-Mg-28, Morgan County, Georgia

Charcoal from Pit 1, just below plow zone, $2 \mathrm{~m}$ diam., $0.7 \mathrm{~m}$ deep at center $\left(33^{\circ} 30^{\prime \prime} \mathrm{N}\right.$ Lat, $83^{\circ} 25^{\prime} \mathrm{W}$ Long). Coll. by Mark Williams, J. R. Caldwell, and Marshall Williams, Lab. Archaeol., Univ. Georgia. Comment (Marshall W.): protohistoric Creek site with busk ceremony trash pit, ca. 50 large (up to $0.5 \mathrm{~m}$ diam.) vessels in whole or in part reconstructed from pit. $\mathrm{C}^{14}$ date agrees with cultural assocs.

\section{UGa-225. Cold Springs Mound, Georgia}

$1550 \pm 65$

Knot from charred log in Cold Spring Mound, Greene Co., Georgia (33 $36^{\prime} 33^{\prime \prime} \mathrm{N}$ Lat, $85^{\circ} 16^{\prime} 20^{\prime \prime} \mathrm{W}$ Long) Survey Test Pit 1, Feature 1. Feature 1 is fired clay area 1.5 to $3.6 \mathrm{~m}, 0.15$ to $0.20 \mathrm{~m}$ below surface of mound summit. Two parallel logs, $2.9 \mathrm{~m}$ apart outlined $\mathrm{E}$ and $\mathrm{W}$ sides of feature, sample is from $\mathrm{W}$ log. Hopewellian site; date compares with other Georgia sites from A.D. 1 to 400. Coll., subm., and comment by Archie Smith, Lab. Archaeol., Univ. Georgia.

\section{UGa-226. Creighton Island conch}

Conch from Site 9-McI-87, S end Creighton I., Georgia (31 $31^{\prime} 7^{\prime \prime}$ $\mathrm{N}$ Lat, $81^{\circ} 20^{\prime} 3^{\prime \prime} \mathrm{W}$ Long) from Pit $1,1.7$ to $1.8 \mathrm{~m}$ below surface in Zone 3. Deposit was from bottom of shell mound and assoc. with decorated fiber-tempered pottery. Coll. and subm. by D. L. Crusoe, Dept. Anthropol., Univ. Georgia. 


\section{UGa-227. Creighton Island oyster}

Oyster from same site at UGa-226, $1.4 \mathrm{~m}$ below surface of Pit 1 . Same pottery assoc. Comment (D.L.C.): 2 dates are reverse of expectation.

\section{Virginia}

Brown Johnson site, Bland Co., Virginia $\left(37^{\circ} 11^{\prime} \mathrm{N}\right.$ Lat, $81^{\circ} 08^{\prime}$ $25^{\prime \prime} \mathrm{W}$ Long). Charcoal samples from a palisaded Late Woodland Indian village.

\section{UGa-176 A. Feature 29}

UGa-176 B.

UGa-179. Feature 13
A.D. 1490

$460 \pm 75$

$430 \pm 90$

A.D. 1520

$735 \pm 75$
A.D. 1215

Comment by collector, H. A. MacCord, Sr., Archaeologist, Commonwealth of Virginia (1971); UGa-176 from bell-shaped storage pit, used as a grave. Date agrees with cultural assocs. UGa-179, from small storage pit, is believed too early as only one occupation of site is evident archaeologically and date A.D. 1500 to 1550 would seem more likely.

\section{Tennessee}

\section{UGa-199. Faust Shelter}

$2335 \pm 65$

385 B.C.

Charcoal from $50 \mathrm{~cm}$ below surface of shelter in Morgan Co. $\left(36^{\circ}\right.$ $11^{\prime} 50^{\prime \prime} \mathrm{N}$ Lat, $84^{\circ} 36^{\prime} 40^{\prime \prime} \mathrm{W}$ Long), subm. by L. L. Loendorf, Univ. Missouri. Comment (L.L.L.): expected date ca. A.D. 0.

\section{Missouri}

UGa-147. Hess site, 23-Mi-55

$355 \pm 75$

\section{A.D. 1595}

Charcoal from Refuse Pit \#3 near center of Structure \#4, a burned Early Mississippian period domicilary structure. (Similar structure date Gak-1309, $350 \pm 90$, Lewis, pers. commun.).

\section{UGa-145. Callahan-Thompson site, 23-Mi-71}

$$
480 \pm 65
$$

Charcoal recovered from just above floor of NE corner of Structure \#1, an Early Mississipian domiciliary structure based on ceramics and nature of occupation.

\section{UGa-148. Callahan-Thompson site, 23-Mi-71}

$570 \pm 90$

Charcoal from Post 91, a charcoal structural support stud inside wall Trench E. Samples from Hess and Callahan-Thompson sites subm. with comments by R. B. Lewis, Dept. Am. Archaeol., Univ. Missouri. 
UGa-244. Towosahgy site

A.D. 1275

Charcoal from Towosahgy State Archaeological site 23-Mi-2 $\left(36^{\circ}\right.$ $41^{\prime} 35^{\prime \prime} \mathrm{N}$ Lat, $89^{\circ} 14^{\prime} 5^{\prime \prime} \mathrm{W}$ Long) from burned post from Stockade A-1. Comment by submitter, J. W. Cottier, Site Archaeologist. Date will help establish occupation of fortified ceremonial centers for Mississippian tradition of SE Missouri.

UGa-243. Towosahgy site

$1060 \pm 260$

Charcoal from same site as UGa-244, Stockade B, with assoc. bastion, a feature not noted in other excavated stockades.

B. West

1. Wyoming

UGa-190. Big Horn Basin

$3860 \pm 75$

1910 в.c.

Chracoal from buried soil in $\mathrm{N}$ Big Horn Basin, Big Horn Co., Wyoming ( $45^{\circ} 0^{\prime} 02^{\prime \prime} \mathrm{N}$ Lat, $108^{\circ} 26^{\prime} 37^{\prime \prime} \mathrm{W}$ Long). Date may represent altithermal interval in area.

UGa-223. Bandit site

$2130 \pm 60$ 180 B.C.

Charcoal from 48-Bh-460, below burned sandstone (44' $81^{\prime} 24^{\prime \prime}$ $\mathrm{N}$ Lat, $108^{\circ} 18^{\prime} 52^{\prime \prime} \mathrm{W}$ Long). Agrees with archaeologic estimate.

2. Montana

UGa-191. Carbon County

$1570 \pm 80$

Charcoal from buried hearth near Montana/Wyoming border $\left(45^{\circ}\right.$ $0^{\prime} 04^{\prime \prime} \mathrm{N}$ Lat, $108^{\circ} 25^{\prime} 44^{\prime \prime} \mathrm{W}$ Long). Hearth has $5 \mathrm{~m}$ overburden. In addition to being a site date, we are also informed as to how fast the overburden builds up in this area, i.e., ca. $1 \mathrm{~m}$ per $300 \mathrm{yr}$.

\section{UGa-192. Big Horn Canyon}

$1920 \pm 65$

Charcoal from small rock shelter along Big Horn Canyon $\left(45^{\circ} 1^{\prime} 5^{\prime \prime}\right.$ N Lat, $108^{\circ} 15^{\prime} 40^{\prime \prime}$ W Long). No cultural assoc.

\section{UGa-193. Carbon County}

$2510 \pm 240$

Small charcoal sample from lowest level in rock shelter $\left(45^{\circ} 3^{\prime} 48^{\prime \prime}\right.$ $\mathrm{N}$ Lat, $108^{\circ} 27^{\prime} 1^{\prime \prime} \mathrm{W}$ Long). Assoc. cultural debris is Angostura; date is younger than expected.

\section{UGa-196. Carbon County}

$1735 \pm 150$

Charcoal from same rock shelter as UGa-193 and -198. Assoc. debris suggests somewhat older age. 
UGa-198. Carbon County

$1690 \pm 60$

Sample from test pit in rock shelter of UGa-193, -196. Agrees well with UGa-196. Wyoming and Montana samples subm. with comments by L. L. Loendorf, Am. Archaeol., Univ. Missouri.

REFERENCES

MacCord, H. A., Sr., 1971, Brown Johnson Site, Bland Co., Virginia: Quarterly Bull., Archeol. Soc. Virginia, v. 25, p. 230-272.

Noakes, J. E. and Brandau, B. L., 1971, University of Georgia radiocarbon dates I: Radiocarbon, v. 13, p. 468-474. 